\title{
Effects of perturbation relative phase on transverse mode instability gain
}

\author{
Michalis N. Zervas *a,b \\ ${ }^{\mathrm{a}}$ Optoelectronics Research Centre, University of Southampton, SO17 1BJ, UK; \\ ${ }^{b}$ Advanced Laser Lab, ORC/SPI Lasers, University of Southampton, SO17 1BJ, Southampton, UK
}

\begin{abstract}
We have shown that the relative phase between the fundamental fiber mode and the transverse perturbation affects significantly the local transverse modal instability (TMI) gain. The gain variation is more pronounced as the core diameter increases. This finding can be used in conjunction with other proposed approaches to develop efficient strategies for mitigating TMI in high power fiber amplifiers and lasers. It also provides some physical insight on the physical origin of the observed large differences in the TMI threshold dependence on core diameter for narrow and broad linewidth operation.
\end{abstract}

Keywords: high power fiber lasers, high power fiber amplifiers, transverse mode instability, phase perturbations.

\section{INTRODUCTION}

Power scaling in single-mode fiber lasers has been hindered by transverse modal instability (TMI) [1]-[13]. In high power operation, the origin of this effect is predominantly thermal and a number of theoretical models have been introduced to explore the nature and investigate the characteristics of TMI. Theoretical models so far consider the interaction of two propagating modes and study the power exchange between the modes and TMI threshold either though STRS static or dynamic mode interactions [2]-[8]. In an alternative approach, TMI has been studied by considering the stability of the amplified fiber fundamental mode (FM) in the presence of transverse perturbations [10],[11]. TMI, along with SRS, pump brightness and bend-induced mechanical reliability, is shown to be limiting the power scalability in high power fiber lasers [13].

Lately, a number of experimental techniques have been suggested for increasing the TMI threshold in high power fiber amplifiers. They include input signal dithering [13] and pump power modulation [14] in order to smear-out the thermally-generated index grating. In an alternative approach, optical lanterns have been used to apply appropriate phase shifts into the excited modes at the output of the lantern and minimize the HOM content, and it has been suggested as a powerful technique to mitigate TMI in HPFAs [15],[16].

In this work, we show theoretically for the first time that differential phase shifts between the FM and the perturbative HOM can affect the overall TMI gain. This new understanding can be used to develop faster control algorithms or better lantern-type input systems with minimum input arms.

\section{THEORETICAL MODEL OUTLINE}

Our TMI analysis is based on the stability analysis of the fundamental mode ( $\left.\mathrm{LP}_{01}\right)$ amplification, in the presence of steady-state heat load and gain saturation [10],[11]. Spatio-temporal perturbations on the $\mathrm{LP}_{01}$ mode $\varepsilon(\mathrm{r}, \varphi, z ; t)$, upper state population $\delta n_{2}(\mathrm{r}, \varphi, \mathrm{z} ; \mathrm{t})$ and temperature change $\delta T_{0}(\mathrm{r}, \varphi, \mathrm{z} ; \mathrm{t})$ are Fourier analyzed as:

$$
\left[\varepsilon, \delta n_{2}, \delta T_{0}\right]^{T}(r, \varphi, z ; t)=J_{m}\left(u_{\varepsilon} r\right) e^{i m \varphi} \int\left[\tilde{\varepsilon}, \delta \tilde{n}_{2}, \delta \tilde{T}\right]^{T}(z ; \Omega) e^{-i \Omega t} d \Omega
$$


The electric field perturbation Fourier components are in general complex numbers $\tilde{\varepsilon}(z ; \Omega)=\tilde{\varepsilon}_{R}(z ; \Omega)+i \tilde{\varepsilon}_{I}(z ; \Omega)$, with the real and imaginary parts expressed as:

$$
\left[\begin{array}{c}
\tilde{\varepsilon}_{R}(z ; \Omega) \\
\tilde{\varepsilon}_{I}(z ; \Omega)
\end{array}\right]=\left[\begin{array}{c}
\varepsilon_{R}(\Omega) \\
\varepsilon_{I}(\Omega)
\end{array}\right] e^{+K(\Omega) z}
$$

Substituting back into the Maxwell's equations for the perturbations [11], we obtain the dispersion relation of the perturbation propagation, giving two propagation constants, namely:

$$
K_{ \pm}=+i \frac{\Omega}{v_{g}} \pm \frac{1}{2} \sqrt{P_{N}^{2}-4 Y_{0}}-\frac{1}{2} P_{N}-\frac{1}{2} \alpha_{\varepsilon}
$$

where $\alpha_{\varepsilon}$ is the additional perturbation propagation loss, $v_{\mathrm{g}}$ is the group velocity, and $P_{\mathrm{N}}, Y_{0}$ are parameters which depend on the local signal \& pump powers, the inversion and thermo-optic fiber parameters. The real and imaginary parts of the transverse perturbation at the output of a segment $\Delta L$ are given by:

$$
\left[\begin{array}{c}
\tilde{\varepsilon}_{R}(\Delta L ; \Omega) \\
\tilde{\varepsilon}_{I}(\Delta L ; \Omega)
\end{array}\right]=c_{p}\left[\begin{array}{l}
\varepsilon_{1 p} \\
\varepsilon_{2 p}
\end{array}\right] \exp \left(+i K_{+} \Delta L\right)+c_{m}\left[\begin{array}{l}
\varepsilon_{1 m} \\
\varepsilon_{2 m}
\end{array}\right] \exp \left(+i K_{-} \Delta L\right)
$$

where $\left[\varepsilon_{1 \mathrm{p}} \varepsilon_{2 \mathrm{p}}\right]^{\mathrm{T}},\left[\varepsilon_{1 \mathrm{~m}} \varepsilon_{2 \mathrm{~m}}\right]^{\mathrm{T}}$ are the corresponding local eigenvectors defining the perturbation local gain as a function of the local signal and pump powers. The constants $c_{\mathrm{p}}$ and $c_{\mathrm{m}}$ depend on the relative phase $\theta_{0}$ between the FM and the perturbation at the input of the segment $\Delta L$ :

$$
\tilde{\varepsilon}(0 ; \Omega)=\tilde{\varepsilon}_{R}(0 ; \Omega)+i \tilde{\varepsilon}_{I}(0 ; \Omega)=\left|\tilde{\varepsilon}_{0}\right| \exp \left(i \theta_{0}\right)
$$

The TMI gain $G_{\mathrm{TMI}}$ is given by:

$$
G_{T M I}(\Delta L ; \Omega)=\frac{|\tilde{\varepsilon}(\Delta L ; \Omega)|^{2}}{\left|\tilde{\varepsilon}_{0}\right|^{2}}
$$

\section{NUMERICAL RESULTS}

Fig. 1 shows the TMI gain for different phase shifts between the fundamental mode and the perturbation as a function of (a) the normalized transverse wavenumber difference, for frequency $=0 \mathrm{~Hz}$ and (b) TMI frequency, for normalized transverse wavenumber difference $\Delta \mathrm{U}=1$ (which corresponds to $U_{\varepsilon}=U_{11}$ ). The other parameters are core/cladding diameters $60 / 240 \mu \mathrm{m}, \mathrm{P}_{\mathrm{s}}=1000 \mathrm{~W}, \mathrm{~g}_{\mathrm{s}}=2.66 \mathrm{~m}^{-1}$. It is shown that the local TMI gain is affected by the relative phase shift 
between the amplified FM and the perturbation. This phase shift can be induced externally (due to the launching conditions, as in [15],[16]) or internally due to non-uniform stresses, heat or inversion distribution.
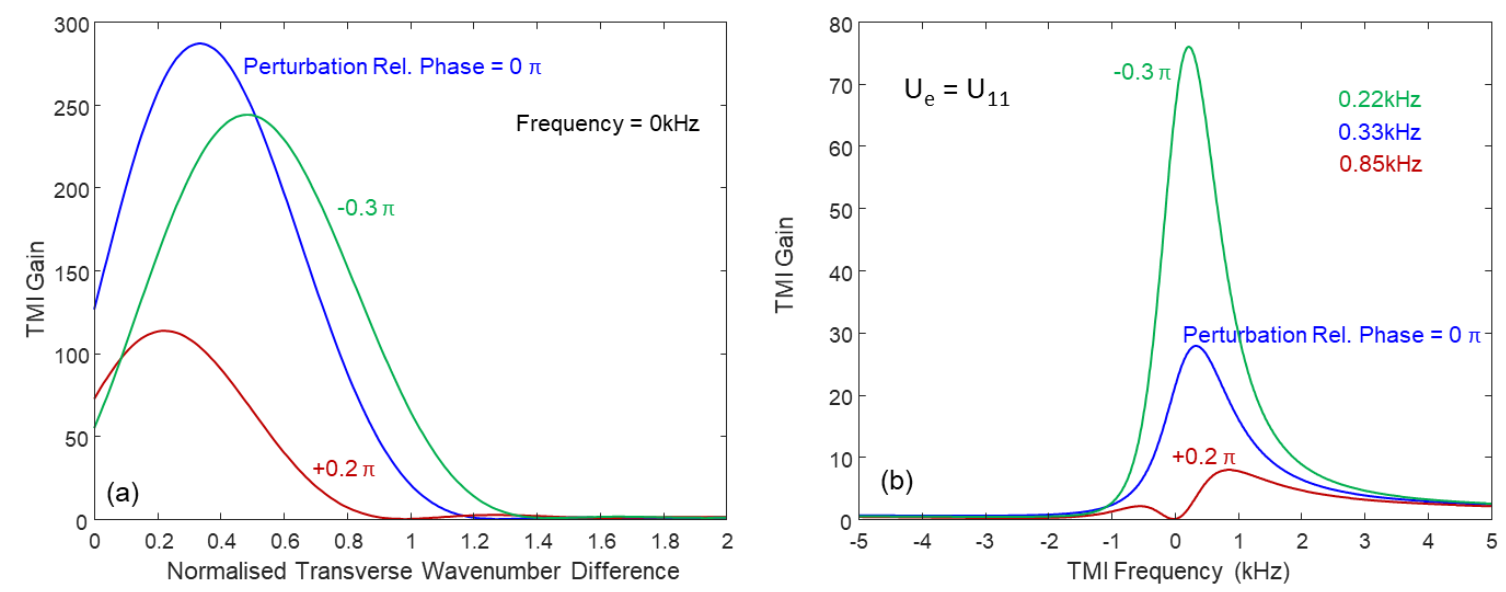

Fig. 1: TMI gain for different phase shifts between fundamental mode and the perturbation as a function of (a) the normalized transverse wavenumber difference (frequency $=0 \mathrm{~Hz})$ and (b) TMI frequency $\left(U_{\varepsilon}=U_{11}\right)$. Core/cladding diameters $60 / 400 \mu \mathrm{m}, \mathrm{P}_{\mathrm{s}}=$ $1000 \mathrm{~W}, \mathrm{~g}_{\mathrm{s}}=2.66 \mathrm{~m}^{-1}$.

It is shown that the local TMI gain is severely affected by the perturbation relative phase and can be substantially decreased or even increased with respect to in-phase response (phase shift $=0$ ). Fig. 2 plots (a) the maximum TMI gain and (b) associated TMI frequency as a function of the phase shift between the fundamental mode and the perturbation, for $U_{\varepsilon}=U_{11}$. The other parameters are: core/cladding diameters $60 / 240 \mu \mathrm{m}$, signal power $\mathrm{P}_{\mathrm{s}}=1000 \mathrm{~W}$ and gain $\mathrm{g}_{\mathrm{s}}=$ $2.66 \mathrm{~m}^{-1}$
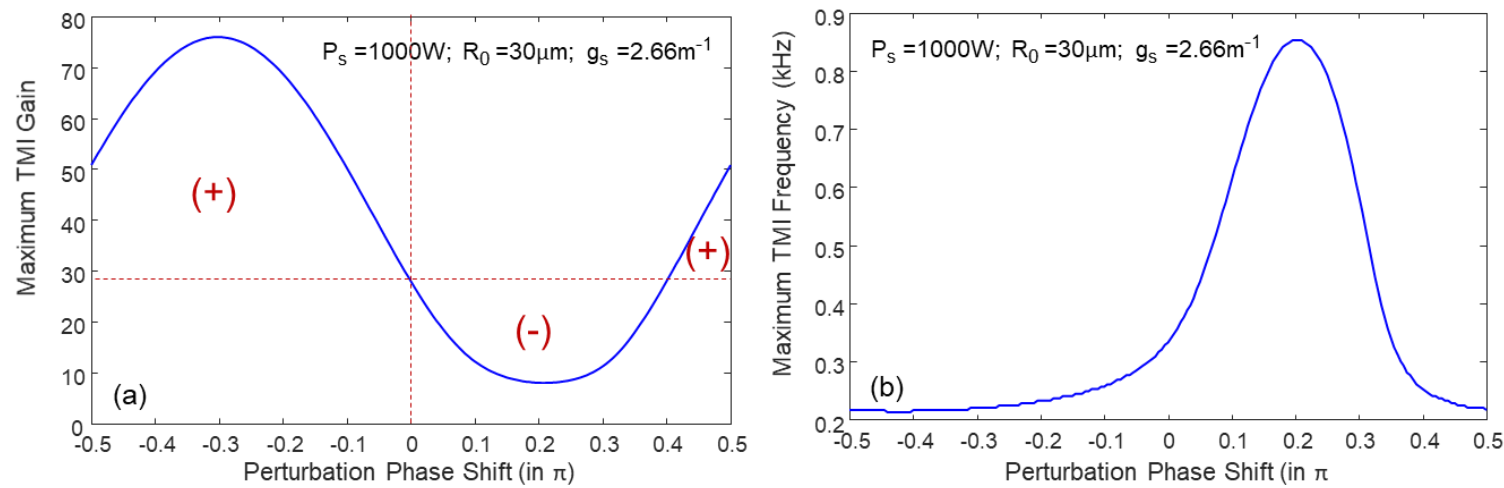

Fig. 2: (a) Maximum TMI gain and (b) associated frequency as a function of the phase shift between fundamental mode and the perturbation, for $U_{\varepsilon}=U_{11}$. Core/cladding diameters $60 / 240 \mu \mathrm{m}, \mathrm{P}_{\mathrm{s}}=1000 \mathrm{~W}, \mathrm{~g}_{\mathrm{s}}=2.66 \mathrm{~m}^{-1}$.

It is shown that the maximum TMI gain is achieved with a perturbation phase shift of $\sim-0.3 \pi$ and frequency of $\sim 220 \mathrm{~Hz}$. This corresponds to $\sim 150 \%$ increase in TMI gain, when compared to the zero phase shift case. The minimum TMI gain, on the other hand, is achieved with a perturbation phase shift of $\sim+0.2 \pi$ and frequency of $\sim 850 \mathrm{~Hz}$, which corresponds to $\sim 60 \%$ decrease in TMI gain, when compared to the zero phase shift case. 
The impact of perturbation phase shift on the maximum TMI gain depends on the core diameter. Fig. 3 plots (a) the maximum TMI gain and (b) associated TMI frequency as a function of the phase shift between the fundamental mode and the perturbation, for different core diameters and $U_{\varepsilon}=U_{11}$. The other parameters are: signal power $\mathrm{P}_{\mathrm{s}}=1000 \mathrm{~W}$ and gain $\mathrm{g}_{\mathrm{s}}=2.66 \mathrm{~m}^{-1}$. This implies that TMI mitigation strategies based on differential phase shift control will be more efficient with larger core fibers.
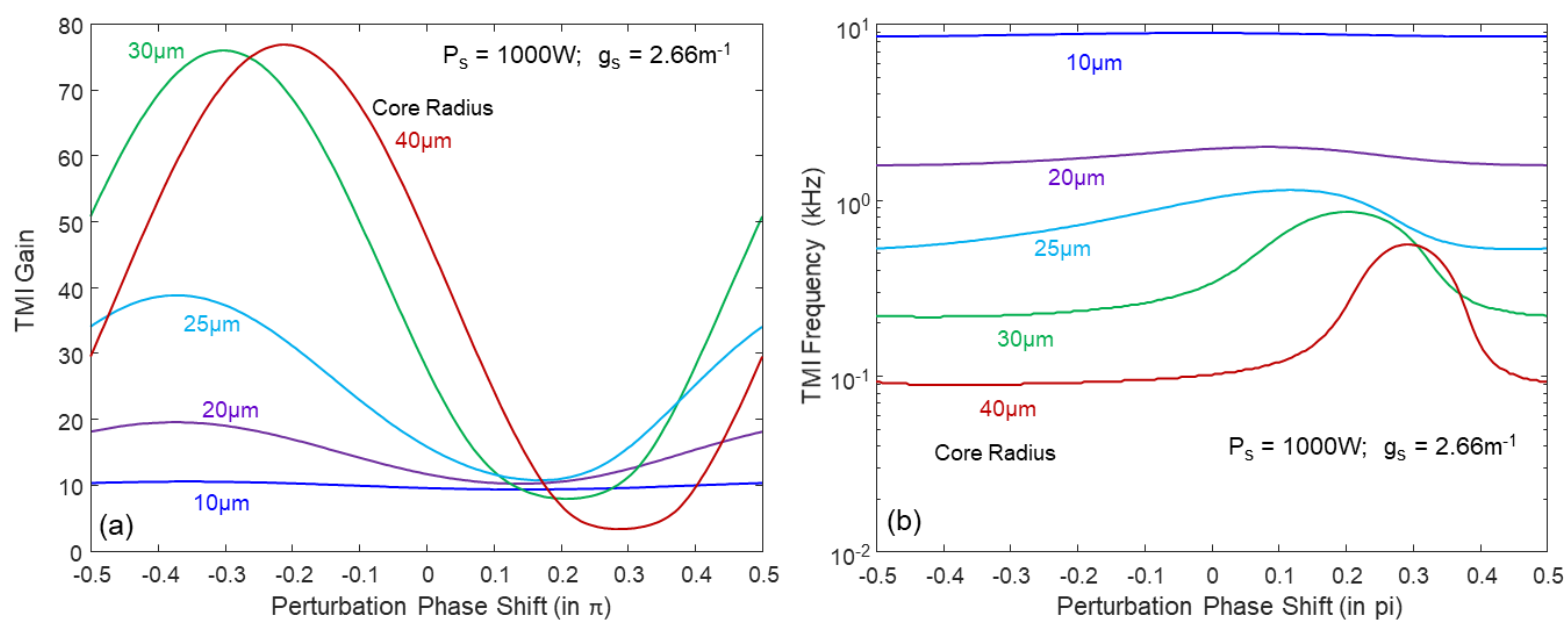

Fig. 3: (a) maximum TMI gain and (b) associated TMI frequency as a function of the phase shift between the fundamental mode and the perturbation, for different core diameters and $U_{\varepsilon}=U_{11} . \mathrm{P}_{\mathrm{s}}=1000 \mathrm{~W}$ and $\mathrm{g}_{\mathrm{s}}=2.66 \mathrm{~m}^{-1}$.

Fig. 4 shows the maximum TMI gain as a function of the phase shift between the fundamental mode and the perturbation, for different signal powers and $U_{\varepsilon}=U_{11}$. The core/cladding diameters $80 / 480 \mu \mathrm{m}$ and gain $\mathrm{g}_{\mathrm{s}}=2.66 \mathrm{~m}^{-1}$. In this case, the corresponding TMI threshold is 400W [11]. It is shown that even at powers well below the nominal TMI threshold $\left(\mathrm{P}_{\mathrm{s}}=300 \mathrm{~W}\right)$, the TMI gain is well above the threshold value when the differential phase shift is $\sim-0.35 \pi$. This dependence on differential phase shift is in very good agreement with recent experimental results [18].

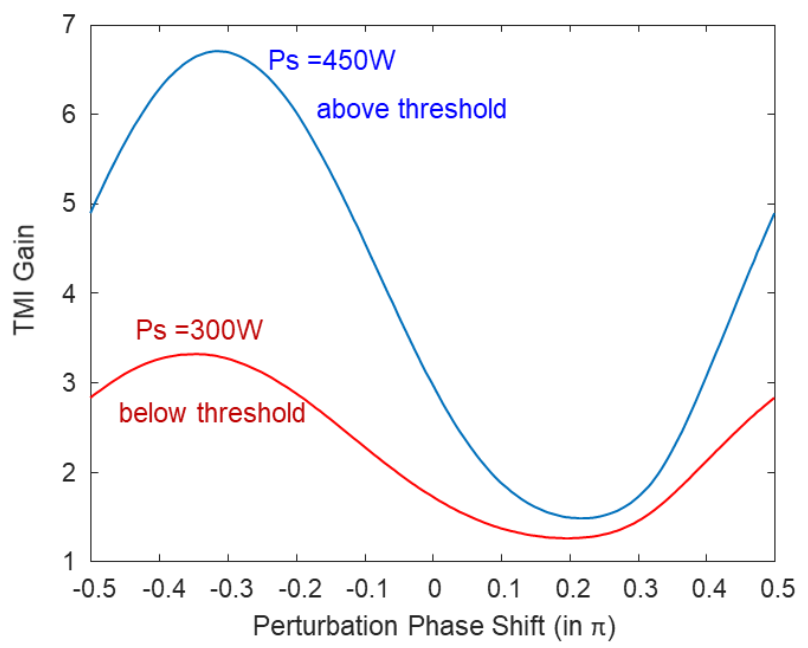

Fig. 4: Maximum TMI gain as a function of the phase shift between the fundamental mode and the perturbation, for different signal powers and $U_{\varepsilon}=U_{11}$. The core/cladding diameters $80 / 480 \mu \mathrm{m}$ and signal gain $\mathrm{g}_{\mathrm{s}}=2.66 \mathrm{~m}^{-1}$. 


\section{SUMMARY}

Power scaling in single-mode fiber lasers has been hindered by transverse modal instability (TMI). In high power operation, the origin of this effect is predominantly thermal and a number of theoretical models have been introduced to explore the nature and investigate the characteristics of TMI. TMI, along with SRS, pump brightness and bend-induced mechanical reliability, is shown to be limiting the power scalability in high power fiber lasers.

In this work, we have shown theoretically for the first time that differential phase shifts between the FM and the perturbative HOM can affect the overall TMI gain. The impact on TMI gain variation depends also on the local signal and pump powers and dopant concentration. It is shown that the local TMI gain can be decreased or even increased with respect to the in-phase response. We have shown that the relative phase between the fundamental fiber mode and the transverse perturbation affects significantly the local TMI gain. The gain variation is more pronounced as the core diameter increases. This is an important finding, which can be used in conjunction with other proposed approaches to develop efficient strategies for mitigating TMI in high power fiber amplifiers and lasers. It also provides some physical insight on the physical origin of the observed large differences in the TMI threshold dependence on core diameter for narrow and broad linewidth operation [11].

\section{Acknowledgements}

This project was supported by the Royal Academy of Engineering under the Research Chairs and Senior Research Fellowships Scheme.

\section{REFERENCES}

[1] Zervas, M. N. and Codemard, C. A., "High Power Fiber Lasers: A Review,” J. Select. Top. Quantum Electron., 20, 0904123 (2014).

[2] Eidam, T., et al, "Experimental observations of the threshold-like onset of mode instabilities in high power fiber amplifiers,”Opt. Express, 1913218 (2011).

[3] Smith, A. V., et al, "Mode instability in high power fiber amplifiers," Opt. Express 19, 10180 (2011).

[4] Jauregui, C., et al, "Physical origin of mode instabilities in high power fiber laser systems," Opt. Express 20,12912 (2012).

[5] Hansen, K. R., et al, "Theoretical analysis of mode instability in high-power fiber amplifiers," Opt. Express 21, 1944 (2013). .

[6] Dong, L., "Stimulated thermal Rayleigh scattering in optical fibers," Opt. Express 21, 2642 (2013).

[7] Naderi, S., et al, "Investigations of modal instabilities in fiber amplifiers through detailed numerical simulations," Opt. Exp. 21, 16111 (2013).

[8] Kuznetsov, M., et al, "Low-threshold mode instability in Yb3+-doped few-mode fiber amplifiers," Opt. Expr. 22, 29714 (2014).

[9] Tao, R., et al, " $1.3 \mathrm{~kW}$ monolithic linearly polarized single-mode master oscillator power amplifier and strategies for mitigating mode instabilities," Photon. Res., 3, 86 (2015).

[10] Zervas, M. N., "TMI Threshold in High Power Fiber Amplifiers," SoW2H.2, OSA Advanced Photonics Congress (2016).

[11] Zervas, M. N., “Transverse Mode Instability Analysis in Fiber Amplifiers,” Proc. SPIE 10083, 100830M (2017).

[12] Zervas, M. N., "Power Scalability in High Power Fiber Amplifiers," CLEO/Europe-EQEC, paper CJ-6.1 (2017).

[13] Zervas, M. N., "Power Scaling Limits in High Power Fiber Amplifiers due to Transverse Mode Instability, Thermal Lensing and Fiber Mechanical Reliability," Proc. SPIE 10512, 10512-04 (2018).

[14] Otto, H.J., et al, "Controlling mode instabilities by dynamic mode excitation with an acousto-optic deflector," Opt. Express 21, 17285 (2013).

[15] Stihler, C., et al, "Controlling mode instabilities at $628 \mathrm{~W}$ average output power in an Yb-doped rod-type fiber amplifier by active modulation of the pump power," Proc. SPIE 10083 100830P (2017). 
[16] Montoya, J., et al, "Photonic lantern adaptive spatial mode control in LMA fiber amplifiers," Opt. Express 24,3405 (2016).

[17] Montoya, J., et al, "Photonic lantern kW-class fiber amplifier," Opt. Express 25, 27543 (2017).

[18] Stihler, C., et al, "Towards the control of the modal energy transfer in transverse mode instabilities," Proc. SPIE $10512,10512-3$ (2018). 